\title{
Generalized Fractional Maxwell Model: Parameter Estimation of a Viscoelastic Material
}

\author{
M. Fernanda P. Costa and C. Ribeiro \\ Department of Mathematics and Applications, Centre of Mathematics, University of Minho, 4710-057 Braga, \\ Portugal
}

\begin{abstract}
In this paper, we show how fractional viscoelastic models can be efficient in the modeling of linear viscoelastic behavior, increasing the fitting accuracy of classic generalized viscoelastic models, such as the Generalized Maxwell model. Experimental data (Loss and Storage modulus in the frequency domain) were retrieved from a Dynamic Mechanical Analysis test considering Carbon Fibre Reinforced Polymer samples. The estimated parameters, for the derived fractional viscoelastic models, were obtained through numerical optimization techniques that minimize the difference between model-predicted values and experimental data. An excellent correlation between analytical and experimental results was observed, minimizing numerical instabilities found on a previous work, for the same experimental setup.
\end{abstract}

Keywords: inverse problem, nonlinear optimization, viscoelasticity, fractional calculus PACS: $02.60 . P n$

\section{INTRODUCTION}

In the work of [1], the relaxation spectra of a Carbon Fibre Reinforced Polymer (CFRP), experimentally obtained through a Dynamic Mechanical Analysis (DMA), was fitted using the classic definition of Storage and Loss modulus given by the Generalized Maxwell Model (GMM) and defined through the following Prony series:

$$
G^{\prime}(\omega)=\mathfrak{R}\left\{G^{*}\right\}=G_{\infty}+\sum_{k=1}^{N} g_{k} \frac{\left(\omega \tau_{k}\right)^{2}}{1+\left(\omega \tau_{k}\right)^{2}}, G^{\prime \prime}(\omega)=\mathfrak{I}\left\{G^{*}\right\}=\sum_{k=1}^{N} g_{k} \frac{\omega \tau_{k}}{1+\left(\omega \tau_{k}\right)^{2}}
$$

where $N$ is the relaxation modes defined by their relaxation strengths $g_{k}$ and their relaxation times $\tau_{k}, k=1,2, \ldots, N$, $G_{\infty}$ is the long term (shear) modulus, $\omega$ is the circular frequency.

As shown in Fig. 1, the optimal fit of the Loss modulus, described by the GMM, did not reproduce with accuracy the experimental data, especially, at high frequencies, exhibiting an oscillatory behavior. It is known, in the literature, that the fractional models are better to describe the behavior of viscoelastic materials [2]. In fact, recent works in this area (see, for example [3]) have shown that fractional order models are a good approximation to the generalized classic viscoelastic models (characterized by adding spring and dashpot elements in series and parallel) when the number of elements tend to infinity. In consequence, generalized fractional viscoelastic models are characterized by a more accurate fit when compared to the classic generalized viscoelastic models. In this work, we aim to validate this statement, by comparing the fitting accuracy of classic viscoelastic models with fractional viscoelastic models. Also, the ability of the Prony series, based on fractional viscoelastic models, to converge with fewer parameters to an optimal value, when compared with classic models, will be highlighted. Our goal is to use fractional models, in particular the Generalized Fractional Maxwell Model (GFMM) as a starting point for the application of inverse analysis. Thus, we firstly present the derivation of the Loss and Storage moduli described by the GFMM. In the next section, we formulate a nonlinear least square problem to estimate the viscoelastic model parameters. Finally, the numerical results and conclusions are presented.

\section{GENERALIZED FRACTIONAL MAXWELL MODEL}

In this section, we present the main equations of the GFMM, namely the complex, storage and loss moduli, that result from replacing integer derivatives by the fractional-derivative time in the differential constitutive equation. The GFMM consists of a Hookean spring in parallel with multiple fractional Maxwell elements (or multiple fractional springpot elements). 
We denote the stress in the spring component by $\sigma_{0}$ and the stress in the first Maxwell element by $\sigma_{1}$, and so on. The total stress $\sigma$ in the generalized model is given by $\sigma=\sigma_{0}+\sigma_{1}+\sigma_{2}+\cdots+\sigma_{N}$ where $\sigma_{0}=G_{\infty} e_{0}(t)$. The constitutive equation for a single fractional Maxwell element is given by [3]

$$
\sigma_{k}(t)+\tau^{\alpha} D_{0^{+}}^{\alpha} \sigma_{k}(t)=g_{k} \tau^{\alpha} D_{0^{+}}^{\alpha} e_{k}(t), \quad k=1, \cdots, N
$$

where $0<\alpha<1$ is the fractional order and the fractional derivative is defined in the Riemann-Liouville form, expressed as [4]

$$
D_{a}^{\alpha} f(x):=\frac{1}{\Gamma(1-\alpha)} \int_{a}^{x} \frac{D f(t)}{(x-t)^{\alpha}} d t=I_{a}^{1-\alpha} D f(x), 0<\alpha<1
$$

where $\Gamma(\alpha)$ denotes the Euler Gamma function, defined for $\Re(\alpha)>0: \Gamma(\alpha)=\int_{0}^{\infty} e^{-u} u^{\alpha-1} d u$. Taking the Laplace transform of Equation (2), applying the respective properties in Fractional Calculus, replacing $s$ by $i \omega$ and rearranging terms, we obtain the expressions

$$
\mathscr{L}\left\{\sigma_{k}(t)\right\}=G_{k}^{*}(i \omega) \mathscr{L}\left\{e_{k}(t)\right\}, G_{k}^{*}(i \omega)=G_{k}^{\prime}(\omega)+i G_{k}^{\prime \prime}(\omega)=\frac{g_{k}\left(i \omega \tau_{k}\right)^{\alpha}}{1+\left(i \omega \tau_{k}\right)^{\alpha}}
$$

where $i=\sqrt{-1}, \mathscr{L}\left\{\sigma_{k}(t)\right\}$ and $\mathscr{L}\{e(t)\}$ denote the Fourier transform of $\sigma_{k}$ and $e_{k}$, respectively.

Combining in parallel $N$ fractional Maxwell elements and one Hookean spring to form the N-parameters of the GFMM, we obtain a so called Prony serie

$$
G^{*}(i \omega)=\frac{\mathscr{L}\{\sigma(t)\}}{\mathscr{L}\{e(t)\}}=G_{\infty}+\sum_{k=1}^{N} \frac{g_{k}\left(\tau_{k} i \omega\right)^{\alpha_{k}}}{1+\left(\tau_{k} i \omega\right)^{\alpha_{k}}},
$$

which is similar to the complex modulus given by the GMM [1]. The real (storage modulus) and imaginary (loss modulus) parts of the GFMM complex modulus (3) can be obtained by summing the respective moduli of individual fractional Maxwell elements

$$
G^{\prime}(\omega)=G_{\infty}+\sum_{k=1}^{N} g_{k} \frac{\left(\omega \tau_{k}\right)^{2 \alpha_{k}}+\left(\omega \tau_{k}\right)^{\alpha_{k}} \cos \frac{\pi \alpha_{k}}{2}}{1+\left(\omega \tau_{k}\right)^{2 \alpha_{k}}+2\left(\omega \tau_{k}\right)^{\alpha_{k}} \cos \frac{\pi \alpha_{k}}{2}}, \quad G^{\prime \prime}(\omega)=\sum_{k=1}^{N} g_{k} \frac{\left(\omega \tau_{k}\right)^{\alpha_{k}} \sin \frac{\pi \alpha_{k}}{2}}{1+\left(\omega \tau_{k}\right)^{2 \alpha_{k}}+2\left(\omega \tau_{k}\right)^{\alpha_{k}} \cos \frac{\pi \alpha_{k}}{2}} .
$$

\section{OPTIMIZATION PROBLEM}

The parameters of the complex modulus $G^{*}(i \omega)$ calculated from the GMM or GFMM are found by fitting functions (1) or (4) to the experimental data, respectively. For the GMM complex modulus $G^{*}(i \omega)$ there are $2 N$ parameters to be estimated, namely, the relaxation times $\tau \equiv\left(\tau_{1}, \ldots, \tau_{N}\right)$ and the relaxation strengths $g \equiv\left(g_{1}, \ldots, g_{N}\right)$. For the GFMM complex modulus $G^{*}(i \omega)$ one additional set of parameters needs to be estimated, that is the order of the fractional derivatives $\alpha \equiv\left(\alpha_{1}, \ldots, \alpha_{N}\right)$.

For each case, the parameters are determined using a nonlinear least squares fit based on the average square of deviation between the predicted values $G^{\prime}\left(\omega_{j}\right), G^{\prime \prime}\left(\omega_{j}\right)$ and the measured $G_{j}^{\prime}, G_{j}^{\prime \prime}$ data at $M$ frequencies $\omega_{j}$. Namely,

$$
\begin{aligned}
\min _{g, \tau \in \mathbb{R}^{N}} F_{1}(g, \tau) \equiv \sum_{j=1}^{M}\left(\left(\frac{G^{\prime}\left(\omega_{j}\right)}{G_{j}^{\prime}}-1\right)^{2}+\left(\frac{G^{\prime \prime}\left(\omega_{j}\right)}{G_{j}^{\prime \prime}}-1\right)^{2}\right) \\
\text { subject to } \quad \begin{array}{ll}
g_{k}>0, \tau_{k}>0, \quad k=1, \ldots, N \\
\tau_{k}<\tau_{k+1}, \quad k=1, \ldots, N-1
\end{array}
\end{aligned}
$$

where $G^{\prime}\left(\omega_{j}\right)$ and $G^{\prime \prime}\left(\omega_{j}\right)$ are calculated values from Equation (1), or

$$
\begin{aligned}
& \min _{g, \tau, \alpha \in \mathbb{R}^{N}} F_{2}(g, \tau, \alpha) \equiv \sum_{j=1}^{M}\left(\left(\frac{G^{\prime}\left(\omega_{j}\right)}{G_{j}^{\prime}}-1\right)^{2}+\left(\frac{G^{\prime \prime}\left(\omega_{j}\right)}{G_{j}^{\prime \prime}}-1\right)^{2}\right) \\
& \text { subject to } \quad 0<\alpha_{k}<1, \quad k=1, \ldots, N \\
& g_{k}>0, \tau_{k}>0, \quad k=1, \ldots, N \\
& \tau_{k}<\tau_{k+1}, \quad k=1, \ldots, N-1
\end{aligned}
$$


where $G^{\prime}\left(\omega_{i}\right)$ and $G^{\prime \prime}\left(\omega_{i}\right)$ are calculated values from Equation (4).

Note that, for the experimental data fitting, we imposed that the relaxation strengths $g_{1}, \ldots, g_{N}$ and the relaxation times $\tau_{1}, \ldots, \tau_{N}$ must be positive, and the relaxation times have to satisfy an ascending order.

\section{NUMERICAL EXPERIMENTS AND CONCLUSIONS}

In [1], $G^{\prime}$ and $G^{\prime \prime}$ experimental data were fitted using the functions in (1), that come from complex modulus $G^{*}(i \omega)$, calculated from GMM. We have used two nonlinear constrained optimization solvers that are available online on the NEOS servers for optimization (http://neos.mcs.anl.gov/neos/solvers). The optimization problem (5) was coded in AMPL - Modeling Language for Mathematical Programming [5]. By default, AMPL applies a presolve phase to the optimization problem, before invoking the solver. Its goal is to reduce the size of the problem sent to the solver. Since the decisions made by the presolve phase rely on numerical computations, sometimes small differences between numbers can have a large effect on the results. Unfortunately, this occurred with the optimization problems (5) and (6). Due to this, we use presolve option 0 to turn off all presolving. We rerun the Ipopt solver from the NEOS for solving the optimization problem (5) again and also to solve the problem (6). The Ipopt is a solver to solve large scale nonlinear optimization problems which implements an interior point method based on a filter line search strategy [6]. As mentioned above, the choice of $N$ is an important issue to obtain the best fit. In the experiments, we test the effect of the number of the terms in the series (1) and (4), on the residual values $F_{1}$ and $F_{2}$, respectively. Table 1 reports the optimal residual values $\left(F_{1}^{*}\right.$ and $F_{2}^{*}$ ) and the CPU time (in sec.), given in columns 2-3 and in columns 4-5, for estimating $2 N$ and $3 N$ parameters (for $N=1,2, \ldots, 15$ ), respectively. This implies to solve fifteen nonlinear constrained least squares problems of type (5) and fifteen problems of type (6). Since we do not have any information about the starting parameters set and their ranges, the starting parameters set was not provided to the Ipopt solver. By default the Ipopt uses the null vector.

TABLE 1. Results obtained by Ipopt

\begin{tabular}{ccccc}
\hline & \multicolumn{2}{c}{ IPOPT } & \multicolumn{2}{c}{ IPOPT } \\
N-Terms Serie & $F_{1}^{*}$ & CPU time [s] & $F_{2}^{*}$ & CPU time [s] \\
\hline 1 & 159.8560 & 0.017 & 80.2011 & 0.065 \\
2 & 71.1437 & 0.062 & 7.5645 & 0.386 \\
3 & 52.9325 & 0.106 & 7.5540 & 1.104 \\
4 & 29.9951 & 0.125 & 7.4935 & 3.098 \\
5 & 20.7001 & 0.316 & 7.5331 & 4.194 \\
6 & 11.2060 & 0.752 & 0.4488 & 1.129 \\
7 & 13.9328 & 0.512 & $\dagger$ & $\dagger$ \\
8 & 3.2850 & 0.838 & 0.3850 & 24.503 \\
9 & 1.9164 & 2.059 & 0.3828 & 39.239 \\
10 & 1.1347 & 2.314 & 0.3689 & 28.163 \\
11 & 0.6708 & 4.335 & 0.3886 & 39.59 \\
12 & 0.4310 & 6.831 & $\dagger$ & $\dagger$ \\
13 & 9.2024 & 10.363 & 0.3850 & 74.603 \\
14 & 10.2686 & 16.277 & $\dagger$ & $\dagger$ \\
15 & $39.9024 \dagger$ & 16.154 & $\dagger$ & $\dagger$ \\
\hline run terminates due to: ‘†’ restoration failed; ‘ $\ddagger$ solved to acceptable level \\
\hline
\end{tabular}

In general, as we can see from Table 1, as the number of terms in the series $(N)$ increases the residual values $F_{1}^{*}$ and $F_{2}^{*}$ decreases up to $N=12$. For values of $N$ greater than 12, it is not possible to further improve the optimal residual values and consequently to obtain a better fit. It is found that the residual decreases more quickly in the optimization problem (6) based on the functions (4) described by GFMM. For example, for $N=6$ the problem has a residual value of 0.4488 , that is only achieved approximately by problem (5) based on the functions (1) described by GMM for $N=12$. When comparing the two models, the GFMM presents the best results. In this case, the best optimal parameter set was found for $N=10$ (see Table 2).

Figure 1(a) and 1(b) plot the storage and loss experimental data vs curve-fit of Loss and Storage of (1) and (4), respectively, using the corresponding best optimal parameter set estimated.

As shown in Fig. 1, the optimized constitutive parameters are not suitable, especially for the Loss modulus described by GMM. As it can be seen from Fig. 1(b), it does not reproduce with accuracy the material's viscoelastic behavior at 
TABLE 2. Optimal parameter set for GFMM $G^{\prime}(\omega)$ and $G^{\prime \prime}(\omega)$.

\begin{tabular}{llllllll}
\hline$k$ & $g_{k}[\mathrm{MPa}]$ & $\tau_{k}[s]$ & $\alpha_{k}$ & $k$ & $g_{k}[\mathrm{MPa}]$ & $\tau_{k}[s]$ & $\alpha_{k}$ \\
\hline 1 & 5147.48 & 0.0636545 & 0.124157 & 6 & $4.97225 \mathrm{e}-05$ & 122324000 & 0.815554 \\
2 & 678.593 & 8579.17 & 0.340389 & 7 & $1.43449 \mathrm{e}-05$ & 122424000 & 0.222429 \\
3 & 1381.39 & 3843980 & 0.999999 & 8 & 292.605 & 756234000 & 0.149816 \\
4 & 5653.46 & 23278400 & 0.999999 & 9 & 2191.13 & 756234000 & 0.999999 \\
5 & 5494.54 & 122274000 & 0.999999 & 10 & 582.678 & 5065390000 & 0.999999 \\
& & & & & & \\
\hline
\end{tabular}

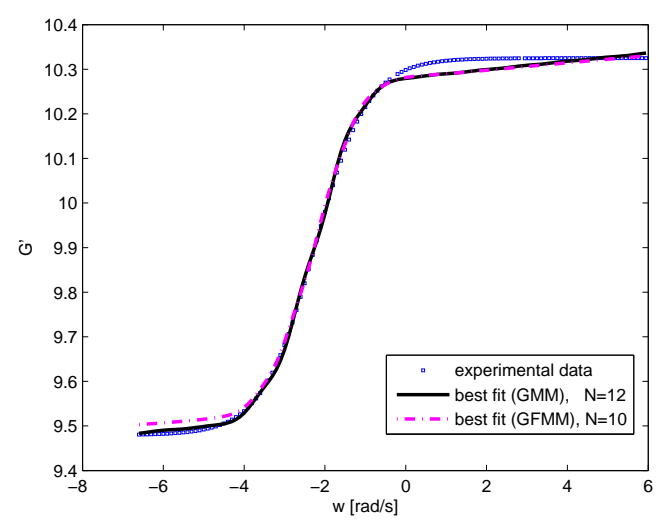

(a) Storage modulus

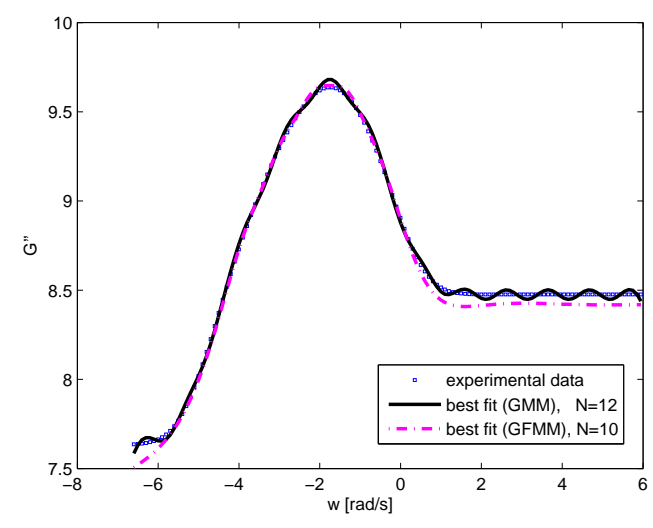

(b) Loss modulus

FIGURE 1. The best fit obtained for the Storage and Loss modulus.

high frequencies, exhibiting an oscillating behavior that has no physical meaning. This drawback is indeed overcome by the Loss modulus described by the GFMM, which leads to a more stable model, especially, for high frequencies.

For future work, the anisotropic behavior of the material will be considered, in order to study the dependence of viscoelastic response on the material orientation and loading direction.

\section{ACKNOWLEDGMENTS}

This work was financed by FEDER funds through COMPETE-Programa Operacional Fatores de Competitividade and by portuguese funds through FCT-Fundação para a Ciência e a Tecnologia within projects PEst-C/MAT/UI0013/2011. We thank the Critical Materials S.A. for making available the DMA experimental data.

\section{REFERENCES}

1. M. Fernanda P. Costa and C. Ribeiro, "Parameter estimation of viscoelastic materials: A test case with different optimization strategies", in International Conference in Numerical Analysis and Applied Mathematics, edited by T.E. Simos et al., AIP Conference Proceedings 1389, 2011, pp. 771-774.

2. H. Schiessel, R. Metzler, T. F. Nonnenmacher and A. Blumen, J. Phys. A: Math. Gen. 28, 6567-6584 (1995).

3. S. W. Katicha and G. W. Flintsch, Rheologica Acta, 1-15 (2012).

4. F. Mainardi, Fractional Calculus and Waves in Linear Viscoelasticity, Imperial College Press, London, 2010, ISBN 978-1-84816-329-4, http: //www.icpress.co.uk/mathematics/p614.html.

5. R. Fourer, D.M. Gay and B. Kernighan, AMPL: A modeling language for mathematical programming, Scientific Press, 1993, ISBN 0-78950-701-3.

6. A. Wächter and L.T. Biegler, Mathematical Programming 106, 25-57 (2006). 